\title{
Genetic heterogeneity in the Freeman-Sheldon syndrome: two adults with probable autosomal recessive inheritance
}

\author{
J S FITZSIMMONS*, V ZALDUA*, AND A R CHRISPIN $\dagger$ \\ From the Clinical Genetic Service*, and the Department of Radiology $\dagger$, City Hospital, Hucknall Road, \\ Nottingham NG5 IPB.
}

SUMmaRY We report two sibs with the clinical features of the Freeman-Sheldon syndrome. The parents were unaffected and consanguineous, suggesting recessive inheritance.

The majority of reported cases of the FreemanSheldon syndrome have been 'sporadic', that is, single cases in families with normal parents. ${ }^{1}$ A number are clearly dominantly inherited ${ }^{2}$ and although Hall et al ${ }^{1}$ quote two instances of recessive inheritance, there has only been one published report of two affected sibs from normal consanguineous parents. $^{3}$ The parents of the affected brother and sister in this report are first cousins. Neither had evidence of the disease and recessive inheritance, in the absence of any other obvious aetiological factors, has been presumed.

Despite the obvious phenotypic differences in the affected subjects, their abnormalities are in keeping with the Freeman-Sheldon syndrome. ${ }^{4}$ Both have involvement of the face with microstomia in association with distal limb contractures and ulnar deviation of the hands: the windmill-vane or windswept deformity. However, there is wide variation in the clinical manifestations of this disease, on occasions causing difficulty in distinguishing it from other conditions associated with distal arthrogryposis. To date, no specific biochemical abnormality has been described and the cause of the joint contractures in this and other similar syndromes is unknown. Many of the clinical features have been considered to result from a disturbance of fetal joint mobility and lack of normal movement early in pregnancy. However, the primary cause of the fetal joint immobility has not always been established and neurogenic or muscular disease, although suggested, cannot always be convincingly demonstrated.

\section{Case reports}

The family pedigree is shown in fig 1 . The parents

Received for publication 30 September 1983. Accepted for publication 21 January 1984.

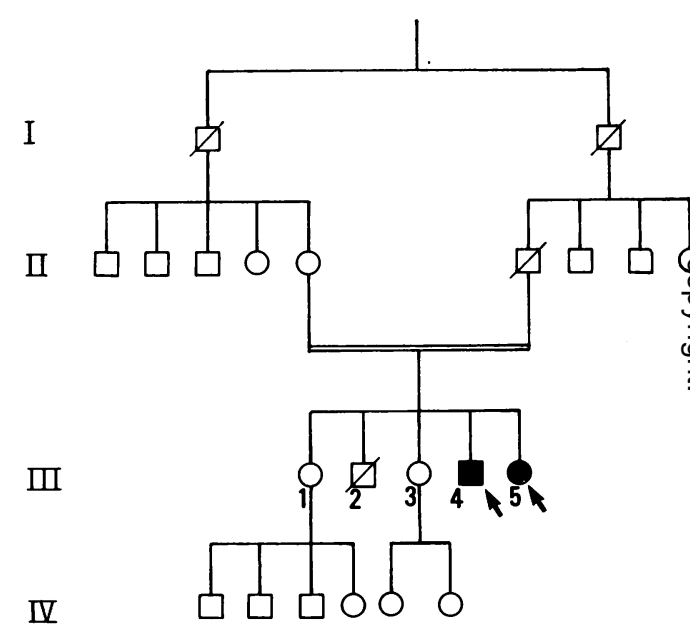

FIG 1 Family pedigree.

are first cousins and had a total of five children. The first born male (III.2) died at the age of 3 weeks, $\frac{O}{3}$ but the cause of death is unknown. III.1 and III.3 are well. There is nothing else of significance and no음 history of congenitally handicapped children born to $>$ other family members. The father, who died aged 68 years from a cerebrovascular accident, was not personally examined but photographs, family com-ments, and information from his medical practitioner $N$ confirmed that he was an active man until his death $\tilde{\omega}$ and did not have any evidence of the disease.

CASE 1 (III.5)

This patient was born on 10.12.50. There was. nothing of note in the pregnancy and her weight was $3062 \mathrm{~g}$ at term. On examination at birth she was noted to have abnormal hands and the thumbs, $\stackrel{\mathbb{\Omega}}{\mathbb{Q}}$ 
which could not be straightened, were adducted across the palms and covered by the flexed fingers. There was also bilateral mild talipes equinovarus and some degree of hypomandibulosis. Despite unexplained feeding difficulties in the first few months, her progress and weight gain were satisfactory. By the age of 8 years, the ulnar deviation of all fingers had progressed to such an extent that operation was necessary and, although this produced some initial improvement, the ulnar deviation and contractures, particularly in the palms, have persisted. During childhood it was noted that she had marked limitation of shoulder movements, a mild scoliosis, and an extremely narrow chest. She had normal vision and hearing but her educational attainments were poor and at the age of 14 years she was admitted to a special school. She left 2 years later, unable to read or write. Her general health has remained good, although in 1978 she developed symptoms of peritonitis and at operation was found to have bilateral salpingitis and an ovarian cyst which was removed. In 1979 she had a left sided pneumothorax which resolved spontaneously. She married at the age of 28 and remains infertile. To date there has been no explanation for this.

On examination in the Genetic Service in 1980 , she had severe contractures of her hands with marked ulnar deviation of all her fingers and facial dysmorphism (fig 2). She had a long immobile face

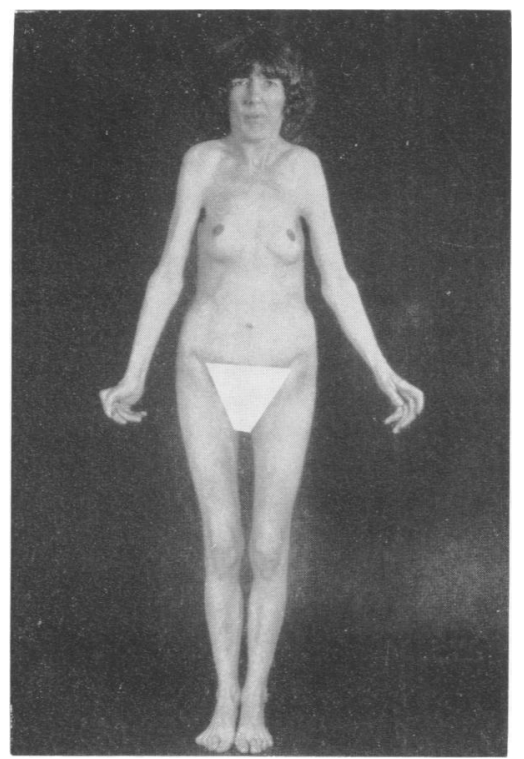

FIG 2 III.5. Narrow upper chest, asthenic appearance, hands severely affected, facial dysmorphism, and axillary pterygia. with a pointed chin, an antimongoloid slant of the palpebral fissures, deep set eyes, narrow nares, and a flattened mid face. Her mouth was small, the oral intercommissural distance measuring $41 \mathrm{~mm}$ (normal $52 \pm 2.5 \mathrm{~mm}^{5}$ ). Her lips were slightly pursed, and below her lower lip there was an $\mathrm{H}$ shaped indentation of the skin. She had deep nasolabial grooves. There was mild webbing of her neck and a low posterior hairline. Although her height, at $154 \mathrm{~cm}$, was on the 10th centile, her weight, at $32.9 \mathrm{~kg}$, was below the 3 rd centile. She had an extremely slender body with thin arms and legs and extremely narrow shoulders. There was marked restriction of shoulder movements and this was associated with pterygia of the anterior and posterior axillary folds. There appeared to be inadequate development of the pectoral muscles and the upper chest was thin and narrow. There was an obvious dislocation of the left radial head. The hips had only approximately $60 \%$ of normal abduction but the knees and ankles appeared normal. She had slight overriding of the toes on the right foot, all of which had a hammer toe deformity. This also affected the toes on the left foot, but there was less marked overriding. There were severe abnormalities of her hands with contractures of the IP and MP/IP joints and marked ulnar deviation of all her fingers. There was loss of normal dermatoglyphs, palmar contractures, and soft tissue webbing between the fingers. There were no abnormalities noted in other

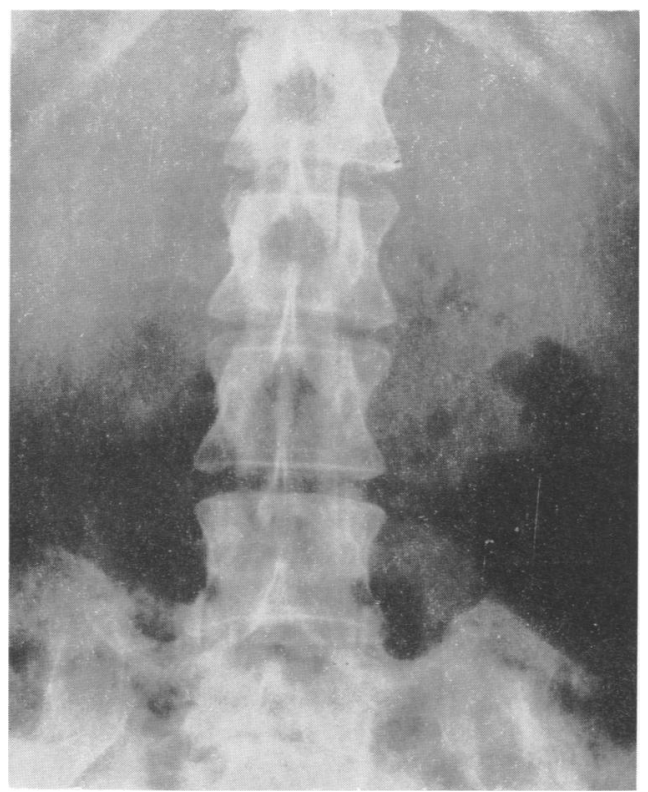

FIG 3 III.5. Tall vertebral bodies and narrow disc spaces. 
systems and despite her extremely narrow chest she had no specific respiratory symptoms. Reflexes in the upper and lower limbs were present, equal, and normal. The karyotype was normal.

\section{Radiology}

The vertebral bodies in the dorsolumbar spine were tall in relationship to their AP diameter. In the lumbar spine the disc spaces looked narrow and the AP diameter of the canal was reduced (fig 3). The interpedicular distances in the lumbar spine did not increase from L1 to L5. There was a spinal curve with the apex on the right in the mid-dorsal region, and a second curve with the apex on the left in the upper dorsal region. The sacrum had lost its usual convexity and had an angle of about $130^{\circ}$ between it and the lumbar spine. The AP diameter

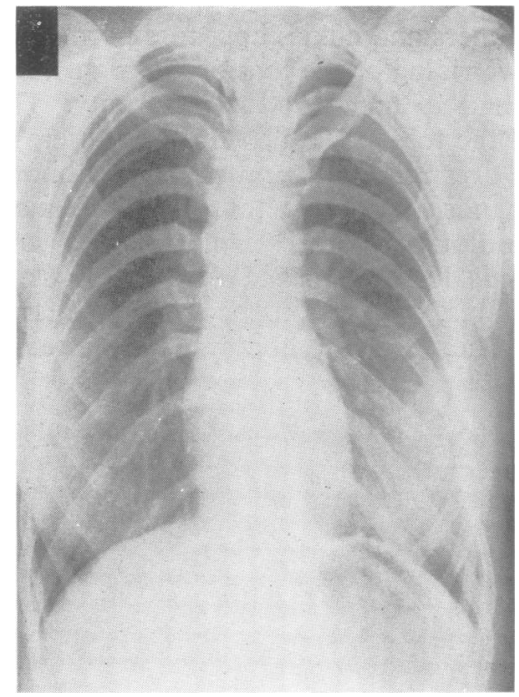

FIG 4 III.5. Reduced transverse diameter of chest with accentuated curvature of the clavicles and scapulae. of the cervical canal narrowed to measure $11 \mathrm{~mm} \frac{\widehat{\Phi}}{\overparen{D}}$ (uncorrected).

The heart and lungs were normal. The ribs in the $\Rightarrow$ dorsal region showed an exceptionally steep forward $\stackrel{\oplus}{+}$ declination (fig 4). The transverse diameter of the chest appeared diminished. The clavicles showed $\overline{\bar{N}}$ accentuated curvature and the scapulae were $\frac{\omega^{\circ}}{\sigma}$ increased in length.

The left elbow showed dislocation of the radial head with a slender proximal end of the radius. The $\vec{O}$ right radius was not dislocated but did not articulate normally with the distal end of the humerus and $\vec{\omega}$ both joints showed poor development of the lateral

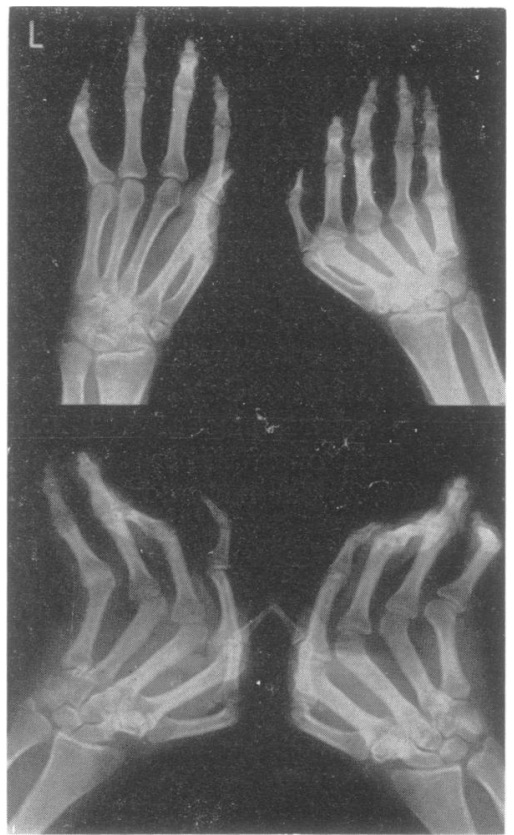

FIG 6 Severe ulnar deviation with windmill-vane deformity of the hands. Top III.5, bottom III.4.

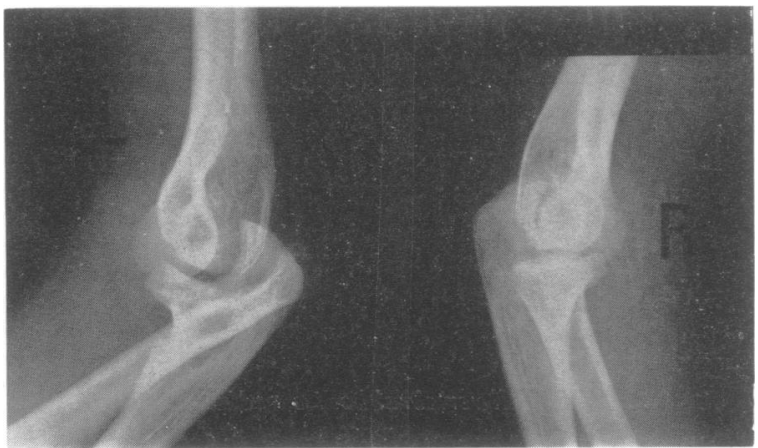

FIG 5 III.5. Dislocation of the left radial head. 
part of the humeral condyles (fig 5). Both hands showed marked ulnar deviation and the carpal centres were smaller than normal (fig 6).

There was absence of the normal angulation of the mandible and the angle between the upper and lower parts was approximately $150^{\circ}$. The maxilla appeared small in relation to the vault.

CASE 2 (III.4)

This patient was born on 8.2.48. There were no details of the pregnancy and his birth weight was unknown. Abnormalities of the feet and hands were noted at birth but there was no history of feeding difficulty. He had a number of operations to correct the severe limb contractures and in addition had operative repair of a right sided squint and a right inguinal hernia. He had no further treatment to his joints after the age of 15 years. Although he did not attempt any examinations at school, he was able to read and write by the time he left. From that time he held down a job as a tractor driver up until the age of 28 .

On examination in the Genetic Clinic in 1983, his height, at $158 \mathrm{~cm}$, was below the $3 \mathrm{rd}$ centile and his weight, $83 \mathrm{~kg}$, above the 97 th centile. In addition to his obvious feet and hand deformities, his right leg was two inches shorter and thinner than his left (fig 7). This caused a limp when he walked and produced a pelvic tilt. His neck appeared short and

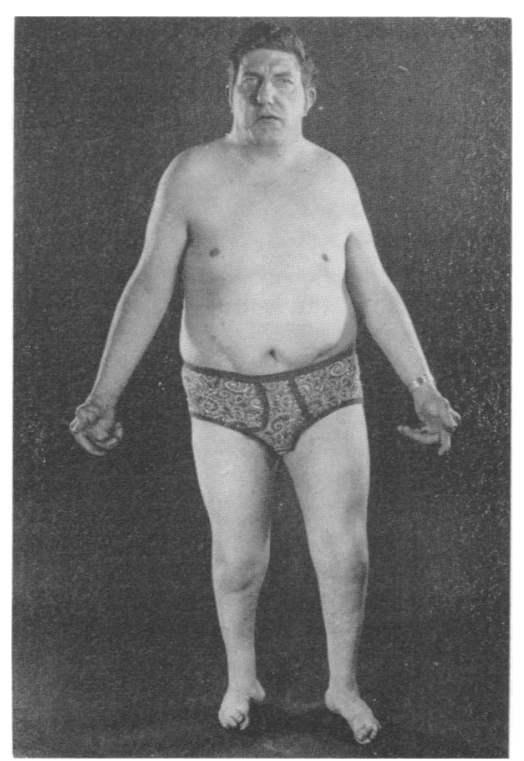

FIG 7 III.4. Obese with short neck, hypoplasia of lower limbs below knees and short right leg, abnormal nipple position, and axillary pterygia. there was definite webbing. He had deep nasolabial furrows, a small mouth, deep set eyes, and skin dimples below his lower lip similar to, but less marked than, those in his sister. His hands showed severe contractures of the IP and MP/IP joints with loss of normal dermatoglyphs and some soft tissue webbing. The middle phalanx of the first finger was extended at the first IP joint, giving an appearance similar to that of rheumatoid arthritis. He had no evidence of dislocation of his elbows but there was marked restriction of shoulder movement and he also had pterygia of the anterior and posterior axillary folds. His nipples appeared excessively low and widely spaced. Although obese, his upper chest appeared deficient in muscle and subcutaneous tissue. His lower limbs showed hypoplasia of the muscles below the knees, more marked on the right than the left. Both feet showed a severe varus deformity with overriding of the first toe onto the big toe, soft tissue syndactyly, and contractures. The right foot was smaller than the left foot.

\section{Radiology}

In the spine the disc height was reduced at the level D12/L1/L2 and there was absence of the usual expansion of the interpedicular distance between $\mathrm{L} 1$ and L5. In the dorsal spine the vertebral bodies appeared square, suggesting a slight increase in height relative to the AP diameter. The dorsal spine was exceptionally straight and had lost its normal kyphosis. The sacrum appeared lower in relation to the ilia. The AP diameter of the cervical canal appeared reduced, measuring $11 \mathrm{~mm}$ minimum (uncorrected).

The hands showed severe ulnar deviation of all fingers with small carpal bones.

In the skull, there was an abnormal angulation of the upper and lower parts of the mandible; the angle was approximately $150^{\circ}$.

\section{Discussion}

In their comprehensive review of the distal arthrogryposes, Hall et al $^{1}$ separated the Freeman-Sheldon syndrome from other sub-groups, but rightly commented on marked phenotypic variation in affected persons. Antley et al $^{6}{ }^{6}$ seeking to identify specific diagnostic criteria, analysed the reports of 34 affected persons and concluded that microstomia, hypoplastic nares, primary telecanthus, and multiple joint contractures were the most significant findings in this syndrome. They recorded the pursed lip or whistling face appearance in only 14 of 26 probands and, although striking in some individuals, this is obviously not a cardinal feature of this syndrome. It was present in mild degree in the female in this 


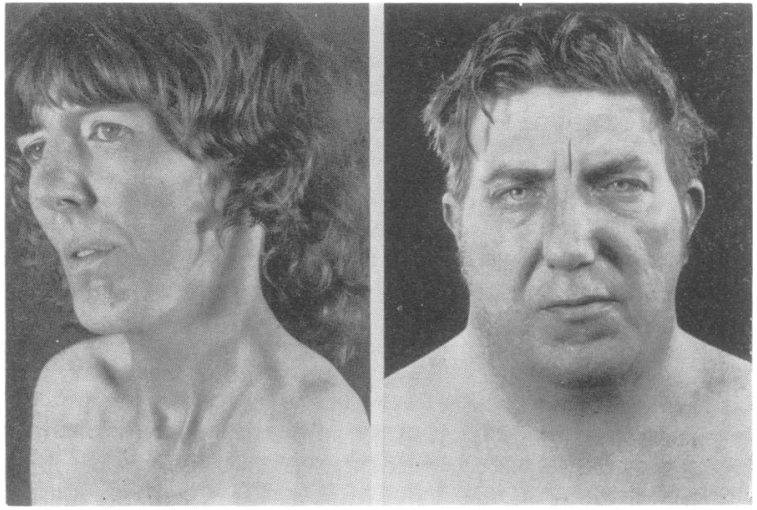

FIG 8 Slight whistling face appearance of III.5 with mid-facial hypoplasia and pursed lips. This is not present in III.4. Both have microstomia and dimpling below the lower lip. report but was absent in her affected brother; both had dimpling of the skin below the lower lip (fig 8). There seems to be little merit in retaining the title whistling face syndrome for this condition, and insistence on the presence of this facial characteristic could obviously result in underdiagnosis of the syndrome.

The basic cause of the immobility of the joints in the hands, feet, and elsewhere remains unclear. Although neurogenic and muscular abnormalities have been reported, the results are far from conclusive and many of the abnormalities noted in these tissues could be accepted as secondary effects of the joint immobility. A widespread abnormality of collagen or other component of connective tissue would seem just as likely and more detailed analysis of connective tissue might be informative. There is no evidence of bone dysplasia and biochemical investigations have been normal. However, the changes in both bone and discs noted in the spinal $x$-rays suggest a significant disturbance in development.

The hands of the two subjects in this report had the classical appearance at birth of the dominant distal arthrogryposis type I described by Hall et al. ${ }^{1}$ The short neck, shortness of stature, immobile facies, scoliosis, and other features are also nonspecific and are described in other types including the dominant type I. There could obviously be difficulty distinguishing the latter from patients with the Freeman-Sheldon syndrome.

Counselling the family of a sporadic case remains difficult. The clinical findings in the patients in this report do not differ significantly from those in families with dominant inheritance or from those patients reported to be sporadic. The latter are difficult to interpret; a number are likely to be recessive in origin and the presence of only one affected person may be a consequence of the social and family effects of an abnormal child. Phenotypic differences are perhaps the most treacherous of all criteria in syndrome differentiation. Hall et al ${ }^{1}$ have made some interesting recommendations for splittingo and delineation of new entities. However, there is $a_{-}$ need for more detailed case reporting and further $O$ discussion on the essential features of some of the? proposed syndromes, including the Freeman-응 Sheldon syndrome. There are undoubtedly con- $\stackrel{\Phi}{?}$ siderable phenotypic similarities between many of $\vec{\bullet}$

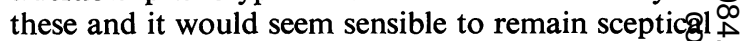
of too much splitting until we have a clearer unde standing of the aetiology and pathology of the diseases.

Our thanks are due to Mr G Gilbert, Medical $\frac{{ }^{\circ}}{\circ}$ Photographer, City Hospital, and the Department of Medical Illustration, Queen's Medical Centre, for 0 the photographs and pedigree. We are also grateful 3 to Miss K Watson for typing the manuscript.

\section{References}

1 Hall JG, Reed SD, Greene G. The distal arthrogryposes:delineation of new entities. Review and nosologic 3 discussion. Am J Med Genet 1982;11:185-239.

2 Frazer FC, Pashayan H, Kadish M. Cranio-carpo-tarsal dysplasia. JAMA 1970;211:1374-6.

3 Alves AF, Azevedo ES. Recessive form of Freeman-o Sheldon's syndrome or 'whistling face'. $J$ Med Genet 1977;14:139-41.

4 Freeman EA, Sheldon JH. Cranio-carpo-tarsal dystrophy. An undescribed congenital malformation. Arch Dis Child 1938;13:277-83.

5 Cervenka J, Figalova P, Gorlin RJ. Cranio-carpo-tarsal dysplasia or the whistling face syndrome. II. Oral intercommissural distance in children. Am J Dis Child 1969; స 117:434-5.

6 Antley RM, Uga N, Burzynski NJ, et al. Diagnostic criteria for the whistling face syndrome. Birth Defects $s$ $1975 ; 11,5: 161-8$.

Correspondence and requests for reprints to $\bigcirc$ Dr J S Fitzsimmons, Clinical Genetic Service, City Hospital, Hucknall Road, Nottingham NG5 1PB. 